\title{
Linear optical properties of one-dimensional Frenkel exciton systems with intersite energy correlations
}

\author{
V. A. Malyshev* \\ GISC, Departamento de Física de Materiales, Universidad Complutense, E-28040 Madrid, Spain
}

A. Rodríguez

GISC, Departamento de Matemática Aplicada y Estadística, Universidad Politécnica, E-20840 Madrid, Spain

F. Domínguez-Adame

GISC, Departamento de Física de Materiales, Universidad Complutense, E-28040 Madrid, Spain

(Received 6 July 1999)

\begin{abstract}
We analyze the effects of intersite energy correlations on the linear optical properties of one-dimensional disordered Frenkel exciton systems. The absorption linewidth and the factor of radiative rate enhancement are studied as a function of the correlation length of the disorder. The absorption line width monotonously approaches the seeding degree of disorder on increasing the correlation length. On the contrary, the factor of radiative rate enhancement shows a nonmonotonous trend, indicating a complicated scenario of the exciton localization in correlated systems. The concept of coherently bound molecules is exploited to explain the numerical results, showing good agreement with theory. Some recent experiments are discussed in the light of the present theory. [S0163-1829(99)07343-9]
\end{abstract}

\section{INTRODUCTION}

Theoretical studies of excited states of quasi-onedimensional (1D) self-assembled molecular systems, such as dipolar coupled molecules in $J$ aggregates, ${ }^{1,2}$ are often based on the well-known Frenkel exciton model $^{3}$ (see Ref. 4 for a comprehensive review). The most remarkable optical properties of $J$ aggregates are the narrowing of their absorption spectra $(J \text { band })^{1,2}$ and the radiative rate enhancement ${ }^{5,6}$ as compared to the monomers, both originating from the collectivization of the local molecular states due to the intermolecular dipolar coupling. The absorption band is narrowed by about the square root of the so-called number of coherently bound molecules $N^{*}$, which, roughly speaking, is the number of molecules covered by the exciton wave function. ${ }^{7}$ The same number determines the factor of radiative rate enhancement. $N^{*}$ is smaller than the physical length of an aggregate since Anderson localization takes place in 1D random systems. ${ }^{8}$ It has been realized that diagonal and off-diagonal disorder are particularly important in determining the optical properties of these systems because $N^{*}$ strongly depends on the degree of disorder. ${ }^{7,9}$

Since the pioneering work by Knapp, ${ }^{7}$ theoretical studies of the optical dynamics of Frenkel excitons in 1D random systems with intersite correlations have attracted much attention. ${ }^{10-14}$ Until recently, however, it was not possible to experimentally prove the occurrence of intersite correlations. Hence, most theoretical descriptions assumed that disorder was indeed uncorrelated. Knoester showed that nonlinear optical techniques are appropriate means to determine both the degree of disorder and the correlation length of the diagonal disorder. ${ }^{10}$ This approach was applied to molecular aggregates of pseudoisocyanine (PIC) by Durrant et al. ${ }^{15}$ and in aggregates of the dye $5,5^{\prime}, 6,6^{\prime}$-tetrachloro-1,1' -diethyl-3, $3^{\prime}$-di(4-sulfobuty)-benzimidazolocarbocianine (TDBC) by Moll et al., ${ }^{16}$ which are known to easily form $J$ aggregates.
One of the general consequences of the occurrence of intersite energy correlations outlined in all the studies concerning the exciton absorption band is the increase of its width as compared to uncorrelated random systems. ${ }^{7,10-14}$ It was found that the absorption line width approaches the seeding degree of disorder on increasing the correlation length of the intersite energy disorder. To the best of our knowledge, however, there was no detailed study of the factor of radiative rate enhancement, carrying out information about the coherently bound molecules. In a recent paper devoted to pairwise correlated diagonal disorder (site energies appear at random to pairs of nearest-neighbor sites), ${ }^{13}$ we found the counterintuitive result that this factor drops upon the occurrence of correlations. This means that excitons at the bottom of the band become more localized after introducing pairwise correlations while the underlying lattice is less disordered, in the sense that only half of the site energies are truly random variables. Stronger localization near the band edge upon introducing the correlations in the disorder was also pointed out in the recent paper by Russ et al. ${ }^{17}$

In this paper, a detailed study of 1D molecular systems with intersite energy correlations is performed in order to uncover the effects of the short-range to long-range correlation length crossover on the linear optical response of Frenkel excitons. We show that the factor of radiative rate enhancement drops upon increasing the energy correlation length whenever this length is smaller than a given characteristic length. For larger energy correlation lengths the behavior of the factor of radiative rate enhancement is the opposite and increases on increasing the correlation length. We provide a simple recipe for estimating this characteristic length. The absorption band width presents a monotonous increase upon rising the energy correlation length and approaches the width of the distribution of seeding disorder when the correlation length exceeds the above mentioned characteristic length. We also use the concept of coherently bound molecules to interpret numerical data. Applying this 
concept in a self-consistent way, we find the scaling of the factor of radiative rate enhancement and the width of exciton absorption line with the energy correlation length and show that theoretical estimates reasonably fit the results of numerical simulations. The remainder of the paper is organized as follows. In Sec. II, we formulate the model we will be dealing with. Section III deals with the theoretical estimates which are then used in Sec. IV for explaining the resutls of numerical simulations. Some recent experiments are discussed in light of the present theory in Sec. V. Section VI summarizes the paper.

\section{MODEL OF DISORDERED SYSTEM}

We consider $N \gg 1$ optically active, two-level molecules forming a regular 1D lattice with spacing unity. To build up our correlated disordered lattice with intersite energy correlation length $N_{c}$, we create $S \equiv N / N_{c}$ consecutive segments with equal transition energies $\epsilon_{s}$ within each segment. The set $\left\{\boldsymbol{\epsilon}_{S}\right\}_{S=1}^{S}$ are statistically independent Gaussian variables with probability distribution

$$
p\left(\epsilon_{s}\right)=\left(\frac{1}{2 \pi \sigma^{2}}\right)^{1 / 2} \exp \left(-\frac{\epsilon_{s}^{2}}{2 \sigma^{2}}\right),
$$

and $\left\langle\epsilon_{s} \epsilon_{s^{\prime}}\right\rangle=\sigma^{2} \delta_{s s^{\prime}}$, where brackets denote the average over the joint energy distribution

$$
P\left(\left\{\epsilon_{s}\right\}\right)=\prod_{s=1}^{S} p\left(\epsilon_{S}\right),
$$

and $\sigma$ is the seeding degree of disorder.

For our present purposes, we will neglect all thermal degrees of freedom (electron-phonon coupling and local lattice distortions) and assume that disorder originates from a static offset of the on-site energies (diagonal disorder). The effective tight-binding Hamiltonian for the 1D Frenkel-exciton problem in the random lattice with correlated disorder can be then written as follows:

$$
\begin{aligned}
\mathcal{H}= & \sum_{s=1}^{S} \epsilon_{s} \sum_{n=1}^{N_{c}}\left|n+N_{c}(s-1)\right\rangle\left\langle n+N_{c}(s-1)\right| \\
& +\sum_{n, m=1}^{N} J_{n m}|n\rangle\langle m|,
\end{aligned}
$$

where $|\nu\rangle$ is the state vector of the $\nu$ th molecule and $J_{n m}$ $=J_{|n-m|}$ are the hopping integrals, which are assumed to be negative, as it takes place for $J$ aggregates, and not subjected to disorder.

We are interested in the absorption line shape and its width as well as in the factor of enhancement of the exciton radiative rate relative to the monomer spontaneous emission rate. The absorption line shape is calculated as

$$
I(E)=\frac{1}{N}\left\langle\sum_{j=1}^{N} \mu_{j}^{2} \delta\left(E-E_{j}\right)\right\rangle .
$$

Here we assume that the system size is much smaller than the optical wavelength. The index $j$ runs over all eigenstates of the Hamiltonian (3) and $E_{j}$ denotes their corresponding eigenenergies. The oscillator strength of the $j$ th eigenstate with components $a_{j}^{(n)}$ is given by

$$
\mu_{j}^{2} \equiv\left(\sum_{n=1}^{N} a_{j}^{(n)}\right)^{2},
$$

where the dipole moment of an isolated monomer is taken to be unity.

The factor of radiative rate enhancement is defined through the average oscillator strength per state at energy $E$ (Ref. 9,18)

$$
\mu_{\mathrm{av}}^{2}(E)=\frac{I(E)}{\rho(E)},
$$

where

$$
\rho(E)=\frac{1}{N}\left\langle\sum_{j=1}^{N} \delta\left(E-E_{j}\right)\right\rangle
$$

is the normalized density of states. We take $\max \left\{\mu_{\mathrm{av}}^{2}(E)\right\}$ as a measure for the enhancement of the exciton radiative rate ${ }^{9}$ or, in other words, for the number of coherently bound molecules $N^{*}{ }^{7}$ In what follows, the above magnitudes will be determined both analytically and numerically as a function of the correlation length $N_{c}$.

\section{THEORETICAL ESTIMATES}

Having presented our model, we now describe the method we have used to estimate the factor of radiative rate enhancement $N^{*}$ and the linear absorption band width $\sigma_{1}$. To this end, we rewrite the Hamiltonian (3) in the excitonic representation using the eigenfunctions of the unperturbed Hamiltonian (with $\epsilon_{s}=0$ for $s=1, \ldots, S$ ). For the sake of simplicity, we assume here periodic boundary conditions. The Bloch plane waves are then the exact eigenfunctions of Eq. (3) in the absence of disorder:

$$
|K\rangle=\frac{1}{\sqrt{N}} \sum_{n=1}^{N} e^{i K n}|n\rangle,
$$

where $K=2 \pi k / N$ belongs to the first Brillouin zone $(0 \leqslant k$ $<N)$. In the $K$ representation, the Hamiltonian (3) reads

$$
\mathcal{H}=\sum_{K} E_{K}|K\rangle\left\langle K\left|+\sum_{K, K^{\prime}} \Delta_{K K^{\prime}}\right| K\right\rangle\left\langle K^{\prime}\right|,
$$

where $E_{K}$ is the unperturbed exciton energy

$$
E_{K}=\sum_{n=1}^{N} J_{n} e^{i K n}
$$

and $\Delta_{K K^{\prime}}$ is the intermode scattering matrix

$$
\Delta_{K K^{\prime}}=\frac{1}{N} \sum_{s=1}^{S} \epsilon_{s} \sum_{n=1}^{N_{c}} e^{i\left(K-K^{\prime}\right)\left[n+N_{c}(s-1)\right]} .
$$

The role of $\Delta_{K K^{\prime}}$ is twofold. The off-diagonal elements $\Delta_{K K^{\prime}}\left(K \neq K^{\prime}\right)$ mix the exciton states. If the typical fluctuation of $\Delta_{K K^{\prime}}$ does not exceed the energy difference between the states $K$ and $K^{\prime}$ (the so called perturbative limit) then the energy shifts of the corresponding states, given by the diag- 
onal elements $\Delta_{K K}$, is the main effect of the disorder and result in inhomogeneous broadening of exciton levels for an ensemble of chains. The typical fluctuation of $\Delta_{K K}$ has a direct relationship with the inhomogeneous width of the exciton state $|K\rangle$.

For nonperturbative magnitudes of the disorder, offdiagonal elements of the scattering matrix $\Delta_{K K^{\prime}}\left(K \neq K^{\prime}\right)$, mix the exciton states, resulting in their localization on chain segments of a typical size smaller than the chain length and subsequently affecting the exciton optical response. Recall that, for a perfect circular chain, only the state $|K=0\rangle$ is coupled to the light and carries the entire exciton oscillator strength, which is then $N$ times larger than that for an isolated molecule. Being mixed with other (nonradiative) states $(K \neq 0)$, the radiative state loses a part of the oscillator strength due to its spreading over the nonradiative ones. Thus, the number of coherently bound molecules $N^{*}<N$ arises as the enhancement factor of the oscillator strength of the localized exciton states. ${ }^{7}$ Accordingly, the inhomogeneous width of the optical exciton line will also be subjected to renormalization since $N$ should be replaced by $N^{*}$.

\section{A. Perturbative treatment}

Assuming the perturbative limit (a quantitative condition of its validity will be given below), let us gain insight into the magnitude of the typical fluctuation of the scattering matrix $\Delta_{K K^{\prime}}$. To this end, one should calculate its second moment using the joint distribution (2) of the seeding fluctuations (being expressed trough linear combinations of Gaussian variables $\epsilon_{s}$ with zero mean, the $\Delta_{K K^{\prime}}$ are also Gaussian variables with $\left\langle\Delta_{K K^{\prime}}\right\rangle=0$ ). The magnitude of our interest will be $\sigma_{K K^{\prime}}^{2} \equiv\left\langle\left|\Delta_{K K^{\prime}}\right|^{2}\right\rangle$. It is an easy task to arrive at

$$
\sigma_{K K^{\prime}}^{2}=\frac{\sigma^{2}}{N N_{c}} \frac{\sin ^{2}\left[\left(K-K^{\prime}\right) N_{c} / 2\right]}{\sin ^{2}\left[\left(K-K^{\prime}\right) / 2\right]} .
$$

In the limits $K, K^{\prime} \ll 1$ and $\left(K-K^{\prime}\right) N_{c} \ll 1$, which are of major importance from the viewpoint of optical properties, Eq. (10) reduces to

$$
\sigma_{K K^{\prime}}^{2}=\sigma^{2} \frac{N_{c}}{N}
$$

This expression generalizes the result we obtained previously for pairwise correlated disorder $\left(N_{c}=2\right)^{13}$ to the case of an arbitrary energy correlation length $N_{c}$. As follows from Eq. (11), the motional narrowing effect $\left(N^{-1}\right.$ scaling) is weakened here by the factor $N_{c}$ and is determined now by the chain length $N$ counted in units of the energy correlation length $N_{c}$ or, in other words, by the number of correlation segments $S=N / N_{c}$. The motional narrowing effect completely disappears at $N_{c}=N$. Similar conclusions were drawn in Refs. 7,9-11.

As it was already mentioned above, the state $|K=0\rangle$ carries the entire oscillator strength of the system so that the optical absorption spectrum presents an isolated Gaussian peak centered around the eigenenergy $E_{K=0}$ and it is characterized by its standard deviation

$$
\sigma_{1}=\sigma \sqrt{\frac{N_{c}}{N}}
$$

This result holds whenever the state $K=0$ does not become mixed with the nearest one $\left|K_{1}\right\rangle$, where $K_{1}=2 \pi / N$. The mixing is governed by the off-diagonal element $\Delta_{K_{1} 0}$. Hence, one should compare $\sigma_{K_{1} 0}$ with the energy difference $E_{K_{1}}-E_{0}$. The perturbative approach is still applicable provided $\sigma_{K_{1} 0}<E_{K_{1}}-E_{0}$ and fails otherwise. Thus, the equality $\sigma_{K_{1} 0}=E_{K_{1}}-E_{0}$ determines a value of $\sigma$ (for fixed both chain length $N$ and energy correlation length $N_{c}$ ) which separates the ranges of perturbative and nonperturbative magnitudes of disorder. Taking $E_{K}$ in the nearest-neighbor approximation

$$
E_{K}=-2 J \cos (K) \simeq-2 J+J K^{2},
$$

where $-J(J>0)$ is the nearest-neighbor coupling and $N$ is assumed to be large, one gets

$$
\sigma=\frac{4 \pi^{2} J}{\left(N^{3} N_{c}\right)^{1 / 2}},
$$

which determines the crossover between the perturbative and nonperturbative limits.

\section{B. Nonperturbative limit}

At nonperturbative magnitudes of disorder, not all molecules of the chain contribute to the optical spectra of the whole ensemble, but only a portion of them being a function of the degree of disorder $\sigma / J$. $^{7}$ The reason is the localization of the excitonic states arising from disorder. Therefore, the number of coherently bound molecules $N^{*}$ (averaged number of molecules covered by optically active localized exciton states) should replace the number of molecules in the system $N$. In Ref. 19, a simple rule for estimating $N^{*}$ was formulated whenever the disorder is uncorrelated. It exploits the findings that (i) the lowest localized exciton states can be classified in several groups of states (two or sometimes three), each one localized on a certain chain segment of a typical size $N^{*}$, (ii) each segment does not overlap with the others $^{19}$ (see also Refs. 20, 21 and Sec. IV for more details) and, what is most important, the states of each group have the energy structure similar to that for a homogeneous chain of size $N^{*}$, i.e., approximately given by Eq. (13) with $N$ replaced by $N^{*}$.

The rule proposed in Ref. 19 for estimating $N^{*}$ consists simply of applying the perturbative criterion (14) to a typical localization segment, i.e., substituting $N$ by $N^{*}$ in Eq. (14) and considering now $N^{*}$ as an unknown parameter. As was shown in Refs. 19, 20, this approach works surprisingly well in fitting the numerical data concerning the optical response of 1D Frenkel excitons. In doing so, one arrives at the following estimate of the number of coherently bound molecules

$$
N^{*}=\frac{1}{N_{c}^{1 / 3}}\left(\frac{4 \pi^{2} J}{\sigma}\right)^{2 / 3}
$$


According to Ref. 7, the standard deviation of the exciton absorption spectrum $\sigma_{1}$ can be estimated by Eq. (12), again replacing $N$ by $N^{*}$, that gives us

$$
\sigma_{1}=N_{c}^{2 / 3} \sigma\left(\frac{\sigma}{4 \pi^{2} J}\right)^{1 / 3} .
$$

The $N_{c}$ scaling laws of $N^{*}$ and $\sigma_{1}$ are the main results of the present paper. Note that $\sigma$-scaling of $N^{*}$ and $\sigma_{1}$ was previously reported in Refs. 9, 19 and 9, 18, 19, 22-24, respectively. We stress that the number of coherently bound molecules drops while the standard deviation of the exciton absorption spectrum rises upon increasing the intersite energy correlations, meaning stronger scattering of the exciton in a more correlated system. This somewhat unexpected result stems from the fact that motional narrowing effect is partially suppressed as intersite energy correlations occurs, yielding a smaller reduction of the seeding disorder as compared to the case of uncorrelated disorder.

\section{Short- to long-range correlations crossover}

The concepts presented in the previous subsection as well as the scaling laws (15) and (16) are valid within the range $N_{c}<N^{*}$. However, both numbers, $N_{c}$ and $N^{*}$, will approach each other as the correlation length $N_{c}$ rises and, starting from a certain value of $N_{c}$ we will obtain $N^{*} \approx N_{c}$. We now provide arguments how to estimate the magnitude of $N_{c}$ at such a crossover. As soon as $N^{*}$ and $N_{c}$ approach each other, there is no reason for the reduction of disorder due to the motional narrowing since the energy of any segment of size $N_{c}$ is fixed, i.e., does not fluctuate. Thus, the typical energy mismatch between two adjacent segments is of the order of $\sigma$. On the other hand, the fact that $N^{*} \approx N_{c}$ means that the coupling between adjacent segments is of the order or smaller than their energy separation $\sigma$. From this it follows that the condition we are looking for is precisely that under which two adjacent segments of size $N_{c}$ start to be decoupled from each other.

In line with this reasoning, let us consider the two segments problem, taking for the sake of simplicity the corresponding Hamiltonian in the nearest-neighbor approximation

$$
\begin{aligned}
\mathcal{H}= & \sum_{\alpha=a, b}\left[\sum_{n=1}^{N_{c}} \epsilon_{\alpha}|\alpha, n\rangle\langle n, \alpha|\right. \\
& \left.-J \sum_{n=1}^{N_{c}-1}(|\alpha, n\rangle\langle n+1, \alpha|+| \alpha, n+1\rangle\langle n, \alpha|)\right] \\
& -J\left(\left|a, N_{c}\right\rangle\langle 1, b|+| b, 1\rangle\left\langle N_{c}, a\right|\right),
\end{aligned}
$$

where $a$ and $b$ are the segment labels and the second term describes the coupling of the segments. Making use of the excitonic transformation

$$
|\alpha, n\rangle=\left(\frac{2}{N_{c}+1}\right)^{1 / 2} \sum_{K} \sin (K n)|\alpha, K\rangle,
$$

where $K=\pi k /\left(N_{c}+1\right)$ and $k$ ranges from 1 to $N_{c}$, one passes from Eq. (17) to

$$
\begin{aligned}
\mathcal{H}= & \sum_{\alpha=a, b} \sum_{K}\left(\epsilon_{\alpha}+E_{K}\right)|\alpha, K\rangle\langle K, \alpha| \\
& +\sum_{K, K^{\prime}}\left(\mathcal{H}_{a b}\right)_{K K^{\prime}}|a, K\rangle\left\langle K^{\prime}, b\right| .
\end{aligned}
$$

Here, $E_{K}$ is given by Eq. (13) and the interaction Hamiltonian reads

$$
\left(\mathcal{H}_{a b}\right)_{K K^{\prime}}=(-1)^{k+1} \frac{2 J}{N_{c}+1} \sin (K) \sin \left(K^{\prime}\right) .
$$

The interaction Hamiltonian $\left(\mathcal{H}_{a b}\right)_{K K^{\prime}}$ couples excitonic modes belonging to different segments and thus leads to their spreading over both segments. Recall that, from the viewpoint of the optical response, mixing of the lowest modes with $K_{1}=K_{1}^{\prime}=\pi /\left(N_{c}+1\right)$ is of major importance. We aim to elucidate for which segment size $N_{c}$ this coupling can be considered as a perturbation. Remembering that the typical energy mismatch between the segments is $\sim \sigma$ and taking $\left|\left(\mathcal{H}_{a b}\right)_{K_{1} K_{1}^{\prime}}\right|=\sigma$, one arrives at the following estimate of the size we are looking for:

$$
N_{c}^{*}+1=\left(\frac{2 \pi^{2} J}{\sigma}\right)^{1 / 3}
$$

where the condition $N_{c} \gg 1$ was used.

Summarizing, the typical energy separation of two adjacent segments starts to dominate over their coupling at $N_{c}$ $>N_{c}^{*}$ so that the segments can be considered as decoupled of each other. As a consequence, the localization length of the lowest exciton mode will be the same that the energy correlation length $N_{c}$.

\section{DISCUSSION OF THE NUMERICAL RESULTS}

In order to calculate the quantities of interest defined by Eqs. (4)-(7) we replaced $\delta\left(E-E_{j}\right)$ by the Heaviside step function $(1 / R) \theta\left(\left|E-E_{j}\right|-R / 2\right)$, where $R$ is the resolution, taken to be 0.05 in all the calculations. For energy units, $J$ $=1$ was chosen. The standard deviation $\sigma$ was also fixed to be 0.2 . The only varied parameter was the energy correlation length $N_{c}$.

We diagonalized the Hamiltonian (3) for open chains of size $N=S N_{c}$, where for a given $N_{c}$, the number $S$ was chosen such that $N$ would be the closest one to 250 . The number of randomly generated systems was 1000 for each value of $N_{c}$. We do not present here the exciton absorption spectra since they are rather standard ones, showing the characteristic asymmetry and red shift due to disorder discussed in detail for uncorrelated disorder in Refs. 9, 18, 25 and for the correlated case in Refs. 7, 11, 13. In Fig. 1, the standard deviation of the absorption spectra $\sigma_{1}$ is shown as a function of the energy correlation length $N_{c}$. To accurately determine the standard deviation $\sigma_{1}$ we fitted the low-energy side of the spectra using Gaussians. One can see from Fig. 1 that $\sigma_{1}$ monotonously increases as $N_{c}$ rises and finally approaches the value $0.2(=\sigma)$ for the case at hand. The crossover, occurring approximately at $N_{c}^{*}=5$, is in good agreement with the estimate $N_{c}^{*} \approx 4$ given by Eq. (20).

Figure 2 shows the behavior of the factor of radiative rate 


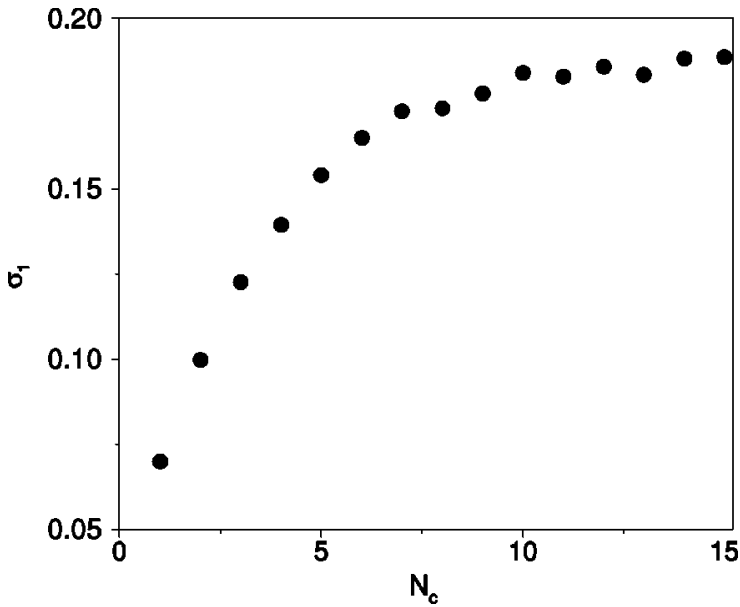

FIG. 1. Standard deviation of the exciton absorption spectra $\sigma_{1}$ versus the energy correlation length $N_{c}$. The degree of seeding disorder is $\sigma=0.2$.

enhancement $\max \left\{\mu_{\mathrm{av}}^{2}(E)\right\}$ as a function of $N_{c}$. First, it drops upon increasing $N_{c}$ and then it grows for $N_{c}>4$, also in full correspondence with our theoretical prediction done in Sec. III. Strong fluctuations observed in Fig. 2 result from the fluctuations in the density of states: the latter appears in the denominator in the definition of $\max \left\{\mu_{\mathrm{av}}^{2}(E)\right\}$ [see Eq. (6)]. We do not present in Fig. 2 how the quantity $\max \left\{\mu_{\mathrm{av}}^{2}(E)\right\}$ behaves for $N_{c}>15$ for several reasons. First, as soon as the segments of correlated energies start to be decoupled of each other [the case for $N_{c}>4$, see Fig. 3(b) and 3(c)], the oscillator strength per state $\mu_{\mathrm{av}}^{2}(E)$ behaves monotonously, i.e., has no maximum (see Appendix A), thus proving to be an inconvenient measure of the extension of the optically active exciton states. Second, it is evident that, for decoupled segments, the localization length $N^{*}$ is equal to the energy correlation length $N_{c}$. Figure 3 confirms the last statement. There, we presented typical realizations of the eight lowest eigenfunctions of the Hamiltonian (3) for three values of the energy correlation length $N_{c}=4,10$, and 40 . They demonstrate that the energy segments actually turn out to be decoupled from each other as the segment size rises.

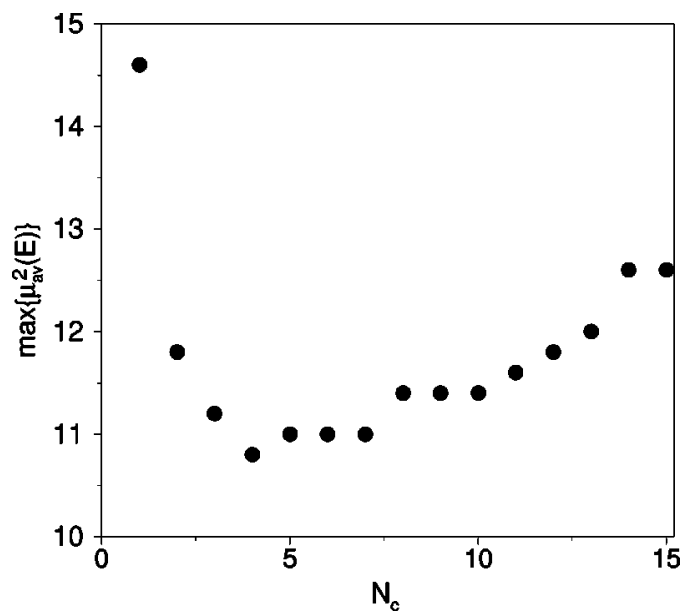

FIG. 2. Factor of the radiative rate enhancement, $\max \left\{\mu_{\mathrm{av}}^{2}(E)\right\}$, versus the energy correlation length $N_{c}$. The degree of seeding disorder is $\sigma=0.2$.
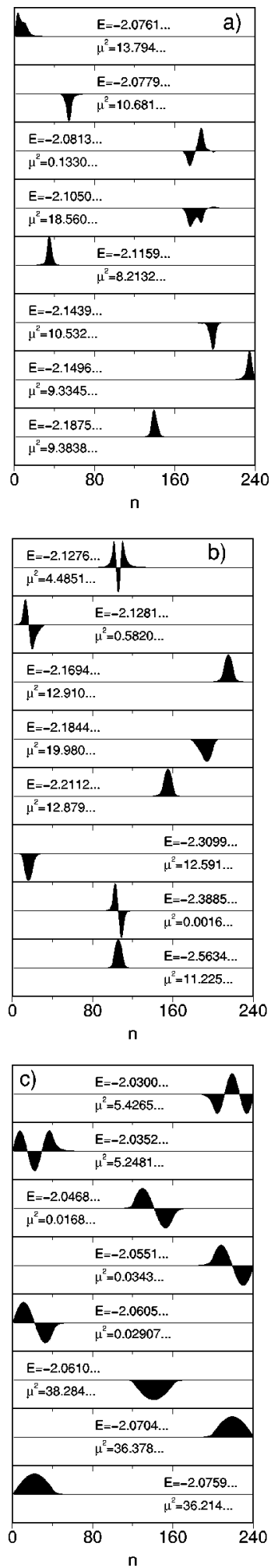

FIG. 3. Typical realizations of the lower exciton eigenmodes for three values of the energy correlation length (a) $N_{c}=4$, (b) 10 , and (c) 40 .

Indeed, for $N_{c}=4$, the typical extension of the eigenfunctions $N^{*}$ of the lowest excitonic states is more than 10 (the oscillator strengths $\mu^{2}$ of nodeless states carry this information). Note that it sufficiently exceeds the energy correlation length. For larger value, $N_{c}=10$, both magnitudes approach each other, although still keeping the relation $N^{*}>N_{c}$. In 
addition, in the last case, the eigenfunctions tend to be grouped on different segments of size $\approx N_{c}$. Finally, for the largest presented value of $N_{c}(=40)$, the eigenfunctions $\varphi_{K n}$, oscillator strengths $\mu_{K}^{2}$ and eigenenergies $E_{K}$ within each group follow fairly well the corresponding formulas for an isolated segment of size $N_{c}$ :

$$
\begin{gathered}
\varphi_{K n}=\left(\frac{2}{N_{c}+1}\right)^{1 / 2} \sin (K n), \\
\mu_{K}^{2}=\frac{1}{2\left(N_{c}+1\right)}\left[1-(-1)^{k}\right]^{2} \cot ^{2}\left(\frac{K}{2}\right), \\
\mathcal{E}_{K}=\epsilon+E_{K},
\end{gathered}
$$

where $\epsilon$ is the site energy of the molecules within each segment. Note that such a structure (called hidden in Ref. 19) of the exciton low-energy spectrum is also present for $N_{c}=4$ but scaled in average by the number $N^{*}$ instead of $N_{c}$.

\section{DISCUSSION OF THE EXPERIMENTAL RESULTS}

In the recent experimental works by Durrant et al. ${ }^{15}$ and by Moll et al. ${ }^{16}$ it was reported on the presence of strong correlations in diagonal disorder in molecular aggregates of PIC and TDBC, respectively. Moreover, it was found in both studies that the energy correlation length $N_{c}$ is of the order of the localization length ${ }^{15}$ and even larger. ${ }^{16}$ This limit strongly suggests that almost no motional narrowing effect has to be detected. ${ }^{10,12}$ Nevertheless, the observed $J$ band width in both cases was definitely motionally narrowed. The possible ordering of the first solvatation shell in the presence of highly ordered aggregate during the freezing process and the subsequent reduction of the local disorder was given as a possible explanation for the $J$ band narrowing.

It seems to be interesting to estimate the critical length $N_{c}^{*}$ for the above aggregates on the basis of the experimental data presented in Refs. 15,16. Taking $J=600$ and $\sigma$ $=13.5 \mathrm{~cm}^{-1}$ for $J$ aggregates of PIC, ${ }^{15}$ one gets $N_{c}^{*} \approx 9$, which is consistent with the relation $N_{c} \simeq N^{*}$ found by Durrant et al. ${ }^{15}$ For $J$ aggregates of $\operatorname{TDBC}(J=850$ and $\sigma$ $=67 \mathrm{~cm}^{-1}$ ), one arrives at $N_{c}^{*} \approx 6$. Thus, the experimental estimation of the energy correlation length $N_{c}$ to be of the order of several hundreds molecules obtained in Ref. 16 contradicts, in our opinion, the magnitude of localization length $N^{*} \approx 40$ extracted from the spectroscopic data.

\section{SUMMARY AND CONCLUDING REMARKS}

In summary, we have studied the effects of intersite energy correlations on the characteristics of linear absorption spectrum corresponding to a weakly localized 1D Frenkel exciton. We have found that the factor of radiative rate enhancement shows a nonmonotonous behavior upon rising $N_{c}$, first decreasing as $N_{c}^{-1 / 3}$ and then growing linearly. The crossover occurs when the typical coupling of segments is smaller than the typical separation between their energy levels, being of the order of the width of the seeding Gaussian energy distribution $\sigma$. The linear trend indicates that the segments become independent one from the other when $N_{c}$ $>\left(2 \pi^{2} J / \sigma\right)^{1 / 3}$. The standard deviation of the absorption line monotonously increases as $N_{c}^{2 / 3}$ for low energy correlation length and saturates when $N_{c}>\left(2 \pi^{2} J / \sigma\right)^{1 / 3}$, namely, when the localization length $N^{*}$ coincides with the energy correlation length $N_{c}$. Our results lead us to a better understanding of recent experimental results in PIC and TDBC molecular aggregates.

\section{ACKNOWLEDGMENTS}

This work was supported by CAM under Project No. 07N/0034/1998. V. A. M. thanks UCM for support under project " Sabáticos Complutense.",

\section{APPENDIX: EVALUATION OF $\mu_{\mathrm{av}}^{2}(E)$ FOR DECOUPLED SEGMENTS}

Let us calculate $\mu_{\mathrm{av}}^{-2}(E)=\rho(E) / I(E)$, assuming the energy segments to be decoupled of each other. Under such an assumption, one can rewrite $\rho(E)$ and $I(E)$ as follows:

$$
\begin{gathered}
\rho(E)=\frac{1}{N}\left\langle\sum_{s=1}^{S} \sum_{k=1}^{N_{c}} \delta\left(E-\epsilon_{s}-E_{K}\right)\right\rangle, \\
I(E)=\frac{1}{N}\left\langle\sum_{s=1}^{S} \sum_{k=1}^{N_{c}} \mu_{K}^{2} \delta\left(E-\epsilon_{s}-E_{K}\right)\right\rangle,
\end{gathered}
$$

where $\quad E_{K}=-2 J \cos [\pi k /(N+1)], \quad k=1,2, \ldots, N_{c} \quad$ and $\left\{\epsilon_{S}\right\}_{S=1}^{S}$ is a stochastic Gaussian energy offset distributed according to Eq. (2). Making use of the integral representation of the $\delta$ function $\delta(x)=(1 / 2 \pi) \int_{-\infty}^{\infty} d t \exp (i x t)$, it is easy to carry out the average in Eqs. (A1a) and (A1b) explicitly to arrive at

$$
\begin{gathered}
\rho(E)=\frac{1}{N_{c}} \sum_{k=1}^{N_{c}}\left(\frac{1}{2 \pi \sigma^{2}}\right)^{1 / 2} \exp \left[-\frac{\left(E-E_{K}\right)^{2}}{2 \sigma^{2}}\right], \\
I(E)=\frac{1}{N_{c}} \sum_{k=1}^{N_{c}} \mu_{K}^{2}\left(\frac{1}{2 \pi \sigma^{2}}\right)^{1 / 2} \exp \left[-\frac{\left(E-E_{K}\right)^{2}}{2 \sigma^{2}}\right] .
\end{gathered}
$$

Accounting further for the fact that the lowest exciton state (with $K_{1}=\pi /\left[N_{c}+1\right]$ ) carries almost the entire oscillator strength $\left(\mu_{K_{1}}^{2} / \Sigma_{K} \mu_{K}^{2}=8 / \pi^{2}\right.$, namely, $\left.81 \%\right)$ and thus keeping in Eq. (A2b) only the first term, we finally get

$$
\mu_{\mathrm{av}}^{-2}(E)=\frac{\pi^{2}}{8 N_{c}}\left\{1+\sum_{k=2}^{N_{c}} \exp \left[\frac{E_{K}-E_{K_{1}}}{\sigma^{2}}\left(E-\frac{E_{K}+E_{K_{1}}}{2}\right)\right]\right\} .
$$

From this it follows that $\mu_{\mathrm{av}}^{-2}(E)$ monotonously rises from the value $\pi^{2} / 8 N_{c}$ to infinity, when passing from $E=-\infty$ to $E=\infty$. As a consequence, $\mu_{\mathrm{av}}^{2}(E)$ monotonously decreases from the value $8 N_{c} / \pi^{2}$ to zero. It should be pointed out that the characteristic energy scale of the exponentials in Eq. (A3) is determined by the ratio $\left(E_{K}-E_{K_{1}}\right) / \sigma^{2}$ which goes down as the segment size $N_{c}$ rises. This means that close to the band bottom, which is precisely the energy region we are interested in, the contribution of the exponentials in Eq. (A3) 
may be large. Then, the magnitudes of $\mu_{\mathrm{av}}^{2}(E)$ obtained from numerical simulations will strongly differ from the asymptotic value $8 N_{c} / \pi^{2}$. Due to the peculiarity outlined above, the oscillator strength per state $\mu_{\mathrm{av}}^{2}(E)$ appears to be

*On leave from All-Russian Research Center "'Vavilov State Optical Institute,' Saint Petersburg, Russia.

${ }^{1}$ E.E. Jelley, Nature (London) 38, 1009 (1936).

${ }^{2}$ G. Scheibe, Angew. Chem. 50, 212 (1937).

${ }^{3}$ J. Frenkel, Phys. Rev. 37, 1276 (1931).

4 A. S. Davydov, Theory of Molecular Excitons (Plenum Press, New York, 1971).

${ }^{5}$ E.G. McRae and M. Kasha, J. Chem. Phys. 28, 721 (1958).

${ }^{6}$ S. De Boer and D.A. Wiersma, Chem. Phys. Lett. 165, 45 (1990).

${ }^{7}$ E.W. Knapp, Chem. Phys. 85, 73 (1984).

${ }^{8}$ N.F. Mott and W.D. Twose, Adv. Phys. 10, 107 (1961).

${ }^{9}$ H. Fidder, J. Knoester, and D.A. Wiersma, J. Chem. Phys. 95, 7880 (1991).

${ }^{10}$ J. Knoester, J. Chem. Phys. 99, 8466 (1993); J. Lumin. 58, 107 (1994).

${ }^{11}$ F. Domínguez-Adame, Phys. Rev. B 51, 12801 (1995).

${ }^{12}$ J. Knoester and F. C. Spano, J Aggregates, edited by T. Kobayashi (World Scientific, Singapore, 1996), p. 111.

${ }^{13}$ F. Domínguez-Adame, V. A. Malyshev, and A. Rodríguez, Chem. Phys. 244, 351 (1999). an inconvenient measure for the extension of the optically active exciton states for the case of larger energy correlation length, when the energy segments are decoupled one from the other.
${ }^{14}$ F. Domínguez-Adame and V. A. Malyshev, J. Lumin. (to be published)

${ }^{15}$ J.R. Durrant, J. Knoester, and D.A. Wiersma, Chem. Phys. Lett. 222, 450 (1994).

${ }^{16}$ J. Moll, S. Daehne, J.R. Durrant, and D.A. Wiersma, J. Chem. Phys. 102, 6362 (1995).

${ }^{17}$ S. Russ, S. Havlin, and I. Webman, Philos. Mag. 77, 1449 (1998).

${ }^{18}$ M. Schreiber and Y. Toyozawa, J. Phys. Soc. Jpn. 51, 1528 (1982); 51, 1537 (1982); 51, 1544 (1982).

${ }^{19}$ V.A. Malyshev, Opt. Spektrosk. 71, 873 (1991) [Opt. Spektrosk. 71, 505 (1991)]; J. Lumin. 55, 225 (1993).

${ }^{20}$ V. Malyshev and P. Moreno, Phys. Rev. B 51, 14587 (1995).

${ }^{21}$ M. Shimizu, S. Suto, T. Goto, A. Watanabe, and M. Matsuda, Phys. Rev. B 58, 5032 (1998).

${ }^{22}$ J. Köhler, A.M. Jayannavar, and P. Reinecker, Z. Phys. B 75, 451 (1989).

${ }^{23}$ A. Tilgner, H.P. Trommsdorff, J.M. Zeigler, and R.M. Hochstrasser, J. Chem. Phys. 96, 781 (1992).

${ }^{24}$ A. Boukahil and D.L. Huber, J. Lumin. 45, 13 (1990).

${ }^{25}$ J. Klafter and J. Jortner, J. Chem. Phys. 68, 1513 (1978). 